\title{
Natural Phenolic Compounds Targeting The AMPK Activation For Metabolic Health
}

\author{
Khoa D.A Nguyen ${ }^{1}$ and Khanh V. Doan ${ }^{2, *}$ \\ ${ }^{1}$ Department of Physiology, ${ }^{2}$ Department of Pharmacology. School of Medicine, Tan Tao University, Duc Hoa, Long An, 850000, Viet Nam., *Corresponding \\ authors: E-mail: khanh.doan@ttu.edu.vn
}

\begin{abstract}
AMP-activated protein kinase (AMPK) is a cellular energy sensor which plays a crucial role in regulation of whole-body energy homeostasis. Activation of AMPK signaling results in favorable effects on mitochondrial function, autophagy, glucose/lipid metabolism, and insulin sensitivity, making it an important therapeutic target in treatment/prevention of metabolic disorders and cancer. Recently, pharmacological studies of natural phenolic compounds indicated that the benefits on metabolic health of these phytochemicals are not only related to their protogenic antioxidant property but also to their AMPK-activating potential. Due to their diverse structures, identification of phenolic compound molecules which have potential to target the AMPK activation for beneficial metabolic effects may be promising in order to develop novel therapeutics in the prevention and/or treatment of metabolic disorders. In this minireview, we summarize beneficial metabolic outcomes of AMPK activation and discuss the capability of natural polyphenols to activate the AMPK pathway focusing on the phenolic acids as potential lead compounds.
\end{abstract}

\section{KEYWORDS}

AMP-activated protein kinase (AMPK), Polyphenol, Phenolic acid, Metabolic benefits.

\section{ARTICLE HISTORY}

Received: 15.08 .2020

Accepted: 15.09 .2020

Online: 01.10 .2020

DOI: https://doi.org/10.53901/fjyu5eg189f

\section{INTRODUCTION}

Obesity, insulin resistance, and dyslipidemia - typical features of "metabolic syndrome" - are strongly associated with globally increasing prevalence of diabetes and cardiovascular disease (CVD). Within the past two decades, our knowledge on molecular biochemical pathways modulating the pathogenesis of diabetes and metabolic syndrome has been expanded. Several novel therapeutic approaches based on the new molecular drug targets within these pathways have been considered, tested, and validated in animals and humans. [1]

AMP-activated protein kinase (AMPK) is a cellular energy sensor which plays a crucial role in regulation of whole-body energy homeostasis [2]. Activation of AMPK signaling results in favorable effects on mitochondrial function, autophagy, glucose/lipid metabolism, and insulin sensitivity, making it an important therapeutic target in treatment/prevention of metabolic disorders and cancer [3, 4]. Recently, pharmacological studies of phytochemicals indicated that benefits on metabolic health of natural polyphenols are not only related to their protogenic antioxidant property but also to their AMPK-activating potential [5].

Polyphenols are a bulky group of small natural molecules which can be categorized into several classes including flavonoids, phenolic acids, stilbenes, and lignans based on the number and presence of phenol rings in their molecule [6]. They are found ubiquitously in plants and possess potent antioxidant activity as well as various bioactivities such as anti-inflammatory, antibacterial, antiproliferative, and anticarcinogenic effects [7]. While metabolic effects and molecular mechanistic action on AMPK pathway of flavonoids and resveratrol (stilbene) were extensively investigated and reviewed [8], the ability to activate AMPK signaling and metabolic actions of other phenolic compounds such as phenolic acids have been just recently explored. In this minireview, we summarize the benefits of AMPK activation on metabolism and discuss the potential for phenolic acids to target this activation and produce beneficial metabolic effects. 


\section{AMP-ACTIVATED PROTEIN KINASE (AMPK) AND ITS AC- TIVATION}

AMPK, a serine/threonine protein kinase, is a sensor of cellular energy status and plays a central role in the regulation of cellular metabolism [9]. It presents in all tissues as a heterotrimeric complex composed of a catalytic $\alpha$ subunit and regulatory $\beta$ and $\gamma$ subunits. Activation of AMPK requires phosphorylation at threonine residue 172 (Thr172) within the activation loop of the $\alpha$ subunit [9]. AMPK is activated by various types of metabolic stresses, drugs, and plant products through the mechanisms involved in the increase of cellular AMP, ADP, or calcium $\mathrm{Ca}^{2+}$ [10]. In mammalian cells, AMPK is activated directly by AMP / ADP or via phosphorylation at Thr172 by the upstream kinases such as iver kinase $B$ 1 (LKB1) or $\mathrm{Ca}^{2+} /$ calmodium-dependent protein kinase kinases (CaMKKs) [10]. During energy stress, ratios of AMP/ATP and/or $\mathrm{ADP} / \mathrm{ATP}$ rise markedly and AMP/ADP compete with ATP to bind to the AMPK $\gamma$ subunit leading to the conformational changes in the kinase domain, which promotes phosphorylation of AMPK Thr172 meanwhile prevents its dephosphorylation [11]. While CaMKKs activate AMPK in response to elevated intracellular $\mathrm{Ca}^{2+}$ independent of changes in adenine nucleotides, LKB1 provides a high basal level of phosphorylation at Thr172 modulated by the binding of AMP to the subunit [11]. Once activated, AMPK in turn phosphorylates and inactivates acetyl-CoA carboxylase (ACC), an important enzyme in fatty acid synthesis. Hence, phosphorylations of AMPK at Thr172 site and the downstream ACC at the major phosphorylated sites are commonly used as indicators for AMPK activation [11].

\section{AMPK ACTIVATION AND METABOLIC BENEFITS}

\section{AMPK activation and lipid metabolism}

Generally, AMPK activation results in the inhibition of fatty acid synthesis while enhancing fatty acid oxidation [12]. By phosphorylating and inhibiting both isoforms of ACC (ACC1 and 2), AMPK activation lowers the concentration of malonyl-CoA, the product of the reaction catalyzed by ACC [13]. Malonyl-CoA serves as an intermediate in the fatty acid synthesis and an inhibitor of carnitine palmitoyl transferase-1, which is involved in fatty acid entry into mitochondria for beta oxidation. In addition, AMPK activation may also enhance fatty acid uptake by inducing the translocation of fatty acid translocase to the plasma membrane by undefined mechanisms [3]. Together with direct and acute effect on ACC, AMPK further inhibits fatty acid synthesis in the longer term by phosphorylating the sterol regulatory element-binding protein $1 \mathrm{c}$ (SREBP-1c, an important transcription factor of lipogenic enzymes) and inhibits cholesterol synthesis by phosphorylating and inactivating the 3-hydroxy-3-methylglutaryl-CoA reductase (HMGR) as well as the phospholipid/triacylglycerol synthesis by inactivating the glycerol phosphate acyl transferase [12].

\section{AMPK activation and mitochondrial biogenesis}

Activated AMPK is known to enhance mitochondrial biogenesis by directly phosphorylating and activating the transcription of peroxisome proliferator-activated receptor gamma co-activator $1 \alpha$ (PGC1 $\alpha$ ), a master transcriptional regulator of genes involved in mitochondrial oxidative metabolism $[3,14]$. PGC- $1 \alpha$ promotes expression of the peroxisome proliferator-activated receptors (PPARs) regulating the genes required for fatty acid oxidation and expression of nuclear respiratory factors (NRFs). NRFs stimulate expression of transcriptional factor A of mitochondria (TFAM), a mitochondrial matrix protein required for mitochondrial DNA replication [15]. Through the direct regulation of PGC-1 $\alpha$ level and transcriptional activity, AMPK activation therefore enhances mitochondrial biogenesis by increasing new mitochondria and expression of nuclear-encoded mitochondrial genes [16]. In addition, PGC1 $\alpha$ can also be up-regulated indirectly through the effect of AMPK activation on sirtuin 1 (Sirt1) which is a nicotinamide adenine dinucleotide (NAD)-dependent deacetylase enzyme that can deacetylate and subsequently increase activity of transcriptional factors [17, 18]. AMPK activation of Sirt1 might be mediated by the up-regulation of NAM-phosphoribosyl transferase (NAMPT), an enzyme that catalyzes the conversion of nicotinamide (NAM) to $\mathrm{NAD}^{+}$, or by altering $\mathrm{NAD}^{+} / \mathrm{NADH}$ ratio independent of NAMPT activity, which ultimately leads to the upregulation of PGC1 $\alpha$ transcriptional activity $[19,20]$.

\section{AMPK activation and autophagy}

Together with increasing mitochondrial biogenesis, AMPK is involved in the disposal of damaged mitochondria and recycling of their contents for reuse as catabolic fuels through a cellular process called autophagy [16]. Autophagy is a "self-eating" process that is induced by nutrient starvation as a mechanism for cell survival [21]. The autophagy-related (Atg) proteins play a crucial role in the core machinery required for autophagosome formation and autophagy induction [22]. AMPK activation stimulates autophagy directly via phosphorylation of autophagy-related proteins in the mTORC1, ULK1 (Unc-51-like autophagy-activating kinase), and lipid kinase complex PIK3C3 (phosphatidylinositol 3-kinase, catalytic subunit type 3) [23, 24]. By recycling components of damaged organelles, AMPK-induced autophagy may play an important role in maintaining overall cellular ATP-generating capacity as the production of new mitochondria during energy starvation demands fuel resources [10]. Moreover, autophagy also regulates the lipid metabolism by preventing accumulation of lipid droplets [25].

\section{AMPK activation, glucose metabolism and insulin sensitivity}

AMPK increases glucose uptake in muscle and adipose tissues via a mechanism that enhances the translocation of glucose transporter 4 (GLUT4) from the cytoplasmic storage vesicles to the plasma membrane [26]. AMPK is also involved in the activation of the plasma membrane-located GLUT1 to promote glucose uptake into other cells [27] and enhances glucose metabolism by increasing mitochondrial biogenesis. In addition, AMPK can further increase insulin sensitivity by enhancing fatty acid oxidation and reducing triglyceride accumulation that is frequently associated with insulin resistance [28]. Consequently, AMPK activation improves insulin sensitivity and serves as a potential therapeutic intervention for the treatment of obesity and type 2 diabetes by regulating glucose and fatty acid metabolism, enhancing mitochondrial function, and clearing the damaged mitochondria through autophagy $[3,4,11$, 29].

\section{PHARMACOLOGICAL AMPK ACTIVATORS}

Physiological conditions of metabolic stresses such as exercise, calorie restriction, and starvation increase the AMP:ATP ratio and hence can physiologically activate the cellular AMPK [30]. In addition to metabolic stresses, it has been shown that AMPK can be activated in response to several pharmacological agents and a number of natural compounds. 


\section{Therapeutic agents as AMPK activators (Biguanide/ Metformin)}

The biguanides have been used for the treatment of type 2 diabetes since the 1950s due to their abilities to inhibit hepatic glucose production and enhance peripheral insulin sensitivity [11]. Although phenformin was subsequently withdrawn due to the life-threatening side effect of lactic acidosis, metformin is still the most widely prescribed biguanide drug for the treatment of type 2 diabetes [31]. Despite their widespread use in clinics, the molecular mechanism of biguanides was first reported in 2000 when metformin was shown to inhibit complex I of the mitochondrial respiratory chain $[32,33]$, which consequently inhibited the mitochondrial ATP synthesis and caused a drop in intracellular ATP levels [11]. Indeed, activation of AMPK by biguanides/ metformin was subsequently demonstrated and the increase in AMP and/or ADP was shown to be required for the AMPK activation induced by biguanides [34, 35]. Although experimental studies revealed that other AMPK-independent mechanisms also contributed to the effect of metformin on hepatic gluconeogenesis suppression, metformin activation of AMPK was demonstrated to play an important in this major therapeutic mode of action of metformin to lower the blood glucose in diabetes [36, 37]. Furthermore, activation of AMPK accounted for the beneficial effect of metformin on insulin sensitivity by stimulating fatty acid oxidation, inhibiting fatty acid synthesis, and reducing triglyceride storage [11].

\section{Thiazolidinediones (TZDs)}

TZDs, another major class of anti-diabetic drugs which include rosiglitazone, pioglitazone, and troglitazone, are known as insulinsensitizing agents including. Although the primary target of TZDs is the nuclear hormone receptor peroxisome proliferatoractivated receptor- (PPAR-), TZDs have also been shown to activate AMPK [38]. Similar to metformin, TZDs indirectly activate AMPK by inhibiting complex 1 of the mitochondrial respiratory chain [39]. In addition increase in adiponectin secretion triggered by PPAR- activation might also involve in AMPK activation induced by TZDs $[40,41]$.

\section{Phytochemicals as AMPK activators}

Various natural products and phytochemicals have been reported to activate AMPK and elicit metabolic benefits in type 2 diabetes and metabolic syndromes. These include resveratrol from red grapes, quercetin from fruits and vegetables, ginsenoside from Panax ginseng, curcumin from Curcuma longa, berberine from Coptis chinensis, epigallocatechin gallate and catechins from green tea, theaflavin from black tea, and hispidulin from snow lotus [11]. Although the structures of these compounds are quite variable, most of them can be classified as polyphenols. Except for the case with salicylate, it seems likely that most of the phytochemicals activate AMPK indirectly by inhibiting mitochondrial ATP production in a similar manner to the biguanides [11]. For example, berberine was shown to inhibit the mitochondrial respiratory complex I whereas resveratrol and quercetin were suggested to inhibit the F1 ATP synthase [42, 43]. This mechanism could be explained for the observation that AMPK activation by resveratrol, berberine, and quercetin was attenuated or abolished in cells expressing the AMP/ADP-insensitive AMPK mutant [11,35]. It is suggested that mitochondrial poisonous effect at low doses of these phytochemicals probably leads to a mild inhibition of mitochondria and thus may be useful to activate AMPK without cellular toxicity [11] (Fig. 1).

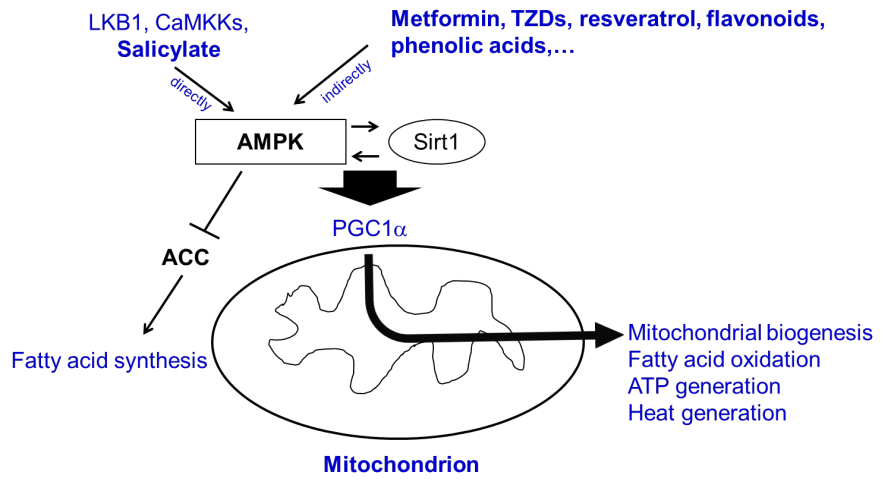

Figure 1 Depicted diagram proposes the mechanistic and beneficial actions of phenolic compounds on AMPK activation<smiles>[R]Oc1cc(O)cc(/C=C/c2ccc(O)cc2)c1</smiles><smiles>O=C(O[C@H]1Cc2c(O)cc(O)cc2O[C@H]1c1cc(O)c(O)c(O)c1)c1cc(O)c(O)c(O)c1</smiles><smiles>COc1cc(/C=C/C(=O)/C=C(O)/C=C/c2ccc(O)c(OC)c2)ccc1O</smiles><smiles>O=c1c(O)c(-c2ccc(O)c(O)c2)oc2cc(O)cc(O)c12</smiles>

Figure 2 Structures of major polyphenols activating the AMPK pathway

\section{Resveratrol and flavonoids}

Resveratrol (3,4',5-trihydroxystilbene, Fig. 2) is found in grapes, red wine, and some berries and has been suggested to give beneficial effect on a variety of illnesses, including obesity/diabetes, cardiovascular disease, and cancer. It has also been suggested to enhance stress endurance and extend lifespan $[8,44]$ probably by mediating some crucial regulators of energy metabolism including AMPK, SIRT1 and PGC $1 \alpha[18,45,46]$. AMPK activation by resveratrol treatment has been observed in many cell types, including muscle cells, hepatocytes and neurons [46, 47]. Resveratrol enhances glucose uptake and mitochondrial biogenesis in muscle, increases mitochondrial biogenesis in the liver, and inhibits hepatic lipid accumulation resulting in improvement of lipid profiles and glucose homeostasis [46, 48, 47]. Resveratrol treatment showed consistent anti-obesity effects by improving lipid and glucose metabolism in diet-induced and genetic obese animal models [8]. However, clinical trials examining the effects of resveratrol on obesity-related energy expenditure, fat oxidation, plasma lipid profiles, and glucose hemostasis produced controversial results $[49,50]$. The lack of resveratrol's effect may be due to the relative short-term intervention and low levels of plasma resveratrol achieved $(1-2 \mu \mathrm{mol} / \mathrm{L})$ compared to the concentrations usually used to activate AMPK in vitro [35]. Thus, although evidence from in vitro, in vivo studies, and limited clinical trials suggests potential anti-obesity effects of resveratrol, the optimal 

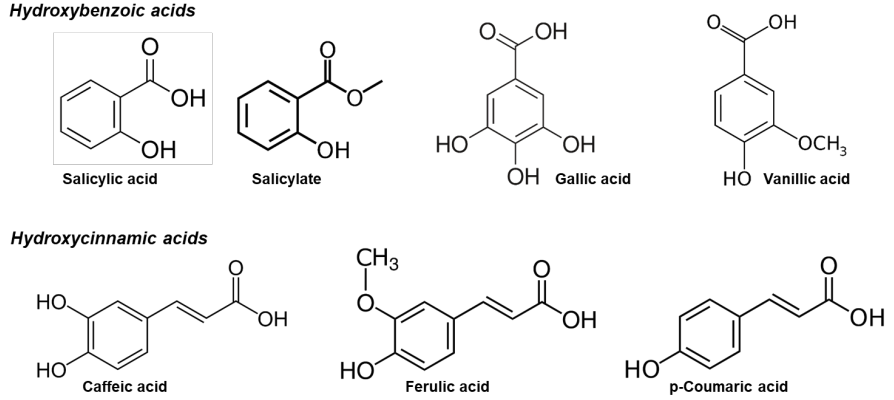

Figure 3 Structures of phenolic acids reported to activate AMPK pathway

doses and study period of dietary supplementation of resveratrol for clinical benefits remain to be determined.

A number of flavonoid molecules have been reported to have potential to activate the AMPK pathway, among which epigallocatechin gallate (EGCG), curcumin and quercetin were extensively studied and reviewed elsewhere [8, 51, 52] (Fig. 2). The metabolic benefits and anti-obesity effects of EGCG, curcumin, and quercetin shown in numerous cell culture and animal studies were identically related to their significant effects on adipogenesis and fatty acid oxidation $[8,51,52,29]$. Specifically, these flavonoid polyphenols showed inhibitory effects on pre-adipocyte differentiation and adipocyte proliferation leading to adipocyte apoptosis and lipogenesis suppression meanwhile promoting lipolysis and fatty acid beta-oxidation [8]. These effects on lipid metabolism could be mediated through AMPK activation which suppressed the expression of PPAR $\gamma$ and CCAAT/enhancer binding protein $\alpha$ $(\mathrm{C} / \mathrm{EBP} \alpha)$, two key regulators of adipocyte differentiation [51, 52]. Remarkably, EGCG was shown to activate AMPK resulting in the suppression of hepatic gluconeogenesis similarly to the action of biguanide [53] while anti-obesity effect of curcumin might be further due to its action on hepatic production of fibroblast growth factor 21 (FGF21) [54]. Moreover, the autophagy induction resulted from AMPK activation leading to reduced lipid accumulation in the liver might also contribute to beneficial effects of these flavonoids on lipid metabolism [55, 56].

\section{AMPK activation by phenolic acids}

Phenolic acids are non-flavonoid polyphenolic compounds which can be classified into two sub-groups: hydroxybenzoic and hydroxycinnamic acids. Hydroxybenzoic acid group includes salicylic, gallic, vanillic, and protocatechuic acids while caffeic, ferulic, $\mathrm{p}$ coumaric, and sinapic acids are hydroxycinnamic acids (Fig. 3). They are found in variety of food plants, often in the bound form. Similar to the flavonoids, phenolic acids possess potent antioxidant activity and have been shown to have various bioactivities including benefits on metabolism [57]. However, their abilities to activate AMPK signaling and regulate metabolic action of phenolic acids have been just recently explored. Except salicylate (salt or ester of salicylic acid) [58], there is a very limited number of studies investigating the potential effects of phenolic acids on AMPK activation.

Salicylic acid and salicylate (its salt or ester) are some of the oldest and most widely used medications which are well-known therapeutic agents in treatment of fever, pain and inflammation. Salicylic acid and salicylate are regarded as plants' cytokine hormones produced to combat against pathogens in response to the infection [15]. The well-defined mechanisms of action of salicylates to inhibit cyclooxygenases (COXs) [59], enzymes catalyzing the prostaglandin synthesis, and NF-kB signaling $[60,61]$ are known for their therapeutic treatment as non-steroidal anti-inflammatory drugs (NSAIDs) in clinical settings. Interestingly, it has been recently shown that salicylate can directly activate the AMPK pathway [62]. The binding of salicylate to AMPK protein was quite similar to AMP molecule, yet its binding site is distinct, causing an allosteric activation of AMPK while inhibiting its Thr172 dephosphorylation. This AMPK activation were demonstrated to account for many metabolic effects of salicylate on glucose/lipid metabolism and cancers $[62,63]$ which cannot be explained by their ability to inhibit COXs and NF-kB signaling. In addition, it has been suggested that the well-known protective effects of aspirin (the acetyl ester of salicylic acid) in various types of cancers could be mediated, at least in part, by AMPK activation [58].

AMPK activation can also be induced by other hydroxybenzoic acids such as gallic, vanillic, and protocatechuic acids. While there was only one in vitro study reporting AMPK activation by protocatechuic acid [64], gallic and vanillic acids were demonstrated to activate AMPK pathway both in vitro and in vivo $[65,66]$. These studies also implicated that AMPK activation plays an important role in mediation of the metabolic beneficial effects of these hydroxybenzoic acids similarly to the case of salicylate $[65,67]$. Thus, these findings together suggest that AMPK activation could be the primary molecular target underlying metabolic benefits of these simple phenolic molecules.

There are increasing evidence showing that other hydroxycinnamic acids such as caffeic, ferulic, and p-coumaric acids can also activate AMPK pathway to exert metabolic actions. For example, caffeic acid and its esters, in addition to target the AMPK pathway in several cancer cell lines $[68,69]$, were shown to activate AMPK in skeletal muscle cells to stimulate glucose uptake and exert potential antidiabetic effects on glucose and lipid metabolism [70, 71]. Similarly, ferulic and p-coumaric acids displayed ability to activate AMPK pathway to regulate metabolic actions in the muscle and adipose cell lines [72, 73]. However, these metabolic effects of hydroxycinnamic acids on AMPK activation were only observed in in vitro models and thus further in vivo validating study is needed.

\section{CONCLUDING REMARK}

Numerous phenolic compounds activating AMPK may be used as useful functional foods or adjuvant therapy designed to improve metabolic health. However, since polyphenol is a broad class of plant secondary metabolites with variable structures, characterization of phenolic molecules targeting the AMPK pathway to improve metabolic homeostasis is an important step to study the relationship between polyphenolic structures and AMPK activation. In this regard, simple phenolic acids emerge as potential lead compounds targeting the AMPK activation to develop new effective therapeutics in prevention/treatment of metabolic diseases.

\section{ACKNOWLEDGEMENT}

This work was supported by the Tan Tao University (TTU) Foundation for Science and Technology Development under grant number TTU.RS.19.305.024.

\section{REFERENCES}

[1] David Moller. New drug targets for type 2 diabetes and the metabolic syndrome. Nature, 414:821-7, 012002. 
[2] David Hardie and Michael Ashford. Ampk: Regulating energy balance at the cellular and whole body levels. Physiology (Bethesda, Md.), 29:99-107, 032014.

[3] Hayley O'Neill, Graham Holloway, and Gregory Steinberg. Ampk regulation of fatty acid metabolism and mitochondrial biogenesis: Implications for obesity. Molecular and cellular endocrinology, 366, 062012.

[4] Kimberly Coughlan, Rudy Valentine, Neil Ruderman, and Asish Saha. Ampk activation: A therapeutic target for type 2 diabetes? Diabetes, metabolic syndrome and obesity : targets and therapy, 7:241-53, 062014.

[5] David Hardie. Regulation of amp-activated protein kinase by natural and synthetic activators. Acta Pharmaceutica Sinica B, 20, 072015.

[6] David Stevenson and Roger Hurst. Polyphenolic phytochemicals - just antioxidants or much more? Cellular and molecular life sciences : CMLS, 64:2900-16, 122007.

[7] Duangjai Tungmunnithum, Areeya Thongboonyou, Apinan Pholboon, and Aujana Yangsabai. Flavonoids and other phenolic compounds from medicinal plants for pharmaceutical and medical aspects: An overview. Medicines (Basel, Switzerland), 5(3):93, Aug 2018. 30149600[pmid].

[8] Shu Wang, Naima Moustaid-Moussa, Lixia Chen, Huanbiao Mo, Anuradha Shastri, Rui Su, Priyanka Bapat, Insook Kwun, and Chwan-Li Shen. Novel insights of dietary polyphenols and obesity. The Journal of nutritional biochemistry, 25:1-18, 01 2014.

[9] David Hardie. Hardie, d. g. amp-activated/snf1 protein kinases: Conserved guardians of cellular energy. nature rev. mol. cell biol. 8, 774-785. Nature reviews. Molecular cell biology, 8:774-85, 112007.

[10] David Hardie, Fiona Ross, and Simon Hawley. Ampk: A nutrient and energy sensor that maintains energy homeostasis. Nature reviews. Molecular cell biology, 13:251-62, 032012.

[11] David Hardie. Ampk: A target for drugs and natural products with effects on both diabetes and cancer. Diabetes, 62:21642172, 072013.

[12] David Hardie. Amp-activated protein kinase: A key regulator of energy balance with many roles in human disease. Journal of Internal Medicine, 276, 052014.

[13] Morgan Fullerton, Sandra Galic, Katarina Marcinko, Sarah Sikkema, Thomas Pulinilkunnil, Zhi-Ping Chen, Hayley O'Neill, Rebecca J Ford, Rengasamy Palanivel, Matthew O'Brien, David Hardie, Stuart Macaulay, Jonathan Schertzer, Jason Dyck, Bryce Denderen, Bruce Kemp, and Gregory Steinberg. Single phosphorylation sites in acc1 and acc 2 regulate lipid homeostasis and the insulin-sensitizing effects of metformin. Nature medicine, 19, 112013.

[14] Sibylle Jäger, Christoph Handschin, Julie St-Pierre, and Bruce Spiegelman. Jager s, handschin c, st-pierre j, spiegelman bm. amp-activated protein kinase (ampk) action in skeletal muscle via direct phosphorylation of pgc-1alpha. proc natl acad sci usa 104: 12017-12022. Proceedings of the National Academy of Sciences of the United States of America, 104:12017-22, 082007.

[15] Jiandie Lin, Christoph Handschin, and Bruce M Spiegelman. Metabolic control through the pgc-1 family of transcription coactivators. Cell metabolism, 1(6):361-370, June 2005.

[16] David Hardie. Amp-activated protein kinase: An energy sensor that regulates all aspects of cell function. Genes development, 25:1895-908, 092011.

[17] Carles Cantó and Johan Auwerx. Pgc- $1 \alpha$, sirt1 and ampk, an energy sensing network that controls energy expenditure.
Current opinion in lipidology, 20:98-105, 052009.

[18] Marie Lagouge, Carmen Argmann, Zachary Gerhart-Hines, Hamid Meziane, Carles Lerin, Frederic Daussin, Nadia Messadeq, Jill Milne, Philip Lambert, Peter Elliott, Bernard Geny, Markku Laakso, Pere Puigserver, and Johan Auwerx. Resveratrol improves mitochondrial function and protects against metabolic disease by activating sirt1 and pgc- $1 \alpha$. Cell, 127:1109-22, 012007.

[19] Marcella Fulco, Yana Cen, Po Zhao, Eric P Hoffman, Michael W McBurney, Anthony A Sauve, and Vittorio Sartorelli. Glucose restriction inhibits skeletal myoblast differentiation by activating sirt1 through ampk-mediated regulation of nampt. Developmental cell, 14(5):661-673, May 2008.

[20] Carles Cantó, Zachary Gerhart-Hines, Jerome Feige, Marie Lagouge, Lilia Noriega, Jill Milne, Peter Elliott, Pere Puigserver, and Johan Auwerx. Ampk regulates energy expenditure by modulating nad+ metabolism and sirt1 activity. Nature, 458:1056-60, 042009.

[21] Daniel Klionsky, Fabio Abdalla, Hagai Abeliovich, Robert Abraham, Abraham Acevedo-Arozena, Khosrow Adeli, Lotta Agholme, Maria Agnello, Patrizia Agostinis, Julio AguirreGhiso, Hyung, Jun Ahn, Ouardia Ait-Mohamed, Slimane AitSi-Ali, Takahiko Akematsu, Shizuo Akira, Hesham Al-Younes, Munir Al-Zeer, Matthew Albert, and Behzad Yeganeh. Guidelines for the use and interpretation of assays for monitoring autophagy. Autophagy, 4454:445-544, 042012.

[22] Yuchen Feng, Ding He, Zhiyuan Yao, and Daniel Klionsky. The machinery of macroautophagy. Cell research, 24, 122013.

[23] Joungmok Kim, Mondira Kundu, Benoit Viollet, and KunLiang Guan. Ampk and mtor regulate autophagy via direct phosphorylation of ulk1. Nature cell biology, 13:132-41, 02 2011.

[24] Daniel Egan, Joungmok Kim, Reuben Shaw, and Kun-Liang Guan. The autophagy initiating kinase ulk1 is regulated via opposing phosphorylation by ampk and mtor. Autophagy, 7:643-4, 062011.

[25] Rajat Singh, Susmita Kaushik, Yongjun Wang, Youqing Xiang, Inna Novak, Masaaki Komatsu, Keiji Tanaka, Ana Maria Cuervo, and Mark J. Czaja. Autophagy regulates lipid metabolism. Nature, 458(7242):1131-1135, April 2009.

[26] Zheng dh, Paul MacLean, Steven Pohnert, John Knight, Ann Olson, William Winder, and G. Dohm. Regulation of muscle glut- 4 transcription by amp-activated protein kinase. Journal of applied physiology (Bethesda, Md. : 1985), 91:1073-83, 102001.

[27] Kay Barnes, Jean C Ingram, Omar H Porras, L Felipe Barros, Emma R Hudson, Lee G D Fryer, Fabienne Foufelle, David Carling, D Grahame Hardie, and Stephen A Baldwin. Activation of glut1 by metabolic and osmotic stress: potential involvement of amp-activated protein kinase (ampk). Journal of cell science, 115(Pt 11):2433-2442, June 2002.

[28] Bradford Lowell and Gerald Shulman. Mitochondrial dysfunction and type 2 diabetes. Science (New York, N.Y.), 307:3847, 022005.

[29] Marc Liesa and Orian Shirihai. Mitochondrial dynamics in the regulation of nutrient utilization and energy expenditure. Cell metabolism, 17:491-506, 042013.

[30] Erik A Richter and Neil B Ruderman. Ampk and the biochemistry of exercise: implications for human health and disease. The Biochemical journal, 418(2):261-275, March 2009.

[31] Maria Mihaylova and Reuben Shaw. The ampk signaling pathway coordinates cell growth, autophagy and metabolism. Nature cell biology, 13:1016-23, 092011. 
[32] Mark OWEN, Elena DORAN, and Andrew HALESTRAP. Evidence that metformin exerts its anti-diabetic effects through inhibition of complex 1 of the mitochondrial respiratory chain. The Biochemical journal, 348 Pt 3:607-14, 072000.

[33] MY El-Mir, V Nogueira, E Fontaine, N Avéret, M Rigoulet, and $X$ Leverve. Dimethylbiguanide inhibits cell respiration via an indirect effect targeted on the respiratory chain complex i. The Journal of biological chemistry, 275(1):223-228, January 2000.

[34] Guangqian Zhou, Robert Myers, Ying Li, Yuli Chen, Xiaolan Shen, Judy Fenyk-Melody, Margaret Wu, John Ventre, Thomas Doebber, Nobuharu Fujii, Nicolas Musi, Michael Hirshman, Laurie Goodyear, and David Moller. Role of amp-activated protein kinase in mechanism of metformin action. The Journal of clinical investigation, 108:1167-74, 112001.

[35] Simon Hawley, Fiona Ross, Cyrille Chevtzoff, Kevin Green, Ashleigh Philp, Sarah Fogarty, Mhairi Towler, Laura Brown, Oluseye Ogunbayo, A Evans, and David Hardie. Use of cells expressing $\gamma$ subunit variants to identify diverse mechanisms of ampk activation. Cell metabolism, 11:554-65, 062010.

[36] Yong Deuk Kim, Keun-Gyu Park, Yong-Soo Lee, Yun-Yong Park, Don-Kyu Kim, Balachandar Nedumaran, Won Gu Jang, Won-Jea Cho, Joohun Ha, In-Kyu Lee, Chul-Ho Lee, and Hueng-Sik Choi. Metformin inhibits hepatic gluconeogenesis through amp-activated protein kinase-dependent regulation of the orphan nuclear receptor shp. Diabetes, 57(2):306-314, 2008.

[37] Russell Miller, Qingwei Chu, Jianxin Xie, Marc Foretz, Benoit Viollet, and Morris Birnbaum. Biguanides suppress hepatic glucagon signaling by decreasing production of cyclic amp. Nature, 494, 012013.

[38] Lee Fryer, Asha Parbu-Patel, and David Carling. The antidiabetic drugs rosiglitazone and metformin stimulate ampactivated protein kinase through distinct signaling pathways. The Journal of biological chemistry, 277:25226-32, 082002.

[39] Barbara Brunmaier, Katrin Staniek, Florian Gras, Nicole Scharf, Aleksandra Althaym, Renate Clara, Michael Roden, Erich Gnaiger, Hans Nohl, Werner Waldhäusl, and Clemens Fürnsinn. Thiazolidinediones, like metformin, inhibit respiratory complex i: A common mechanism contributing to their antidiabetic actions? Diabetes, 53:1052-9, 042004.

[40] Tetsuya Kubota Hiroki Kumagai Shinsuke Itoh Hidemi Satoh Wataru Yano Hitomi Ogata Kumpei Tokuyama Iseki Takamoto Tomoka Mineyama Michiro Ishikawa Masao Moroi Kaoru Sugi Toshimasa Yamauchi Kohjiro Ueki Kazuyuki Tobe Tetsuo Noda Ryozo Nagai Takashi Kadowaki Naoto Kubota, Yasuo Terauchi. Pioglitazone ameliorates insulin resistance and diabetes by both adiponectin-dependent and -independent pathways. The Journal of Biological Chemistry 281, 8748-8755, 012006.

[41] Masato Iwabu, Toshimasa Yamauchi, Miki Okada-Iwabu, Koji Sato, Tatsuro Nakagawa, Masaaki Funata, Mamiko Yamaguchi, Shigeyuki Namiki, Ryo Nakayama, Mitsuhisa Tabata, Hitomi Ogata, Naoto Kubota, Iseki Takamoto, Yukiko Hayashi, Naoko Yamauchi, Hironori Waki, Masashi Fukayama, Ichizo Nishino, Kumpei Tokuyama, and Takashi Kadowaki. Adiponectin and adipor1 regulate pgc-1 and mitochondria by ca2+ and ampk/sirt1. Nature, 464:1313-9, 03 2010.

[42] Jonathan R. Gledhill, Martin G. Montgomery, Andrew G. W. Leslie, and John E. Walker. Mechanism of inhibition of bovine f1-atpase by resveratrol and related polyphenols. Proceedings of the National Academy of Sciences, 104(34):13632-13637, 2007.

[43] Nigel Turner, Jing-Ya Li, Alison Gosby, Sabrina W.C. To, Zhe Cheng, Hiroyuki Miyoshi, Makoto M. Taketo, Gregory J. Cooney, Edward W. Kraegen, David E. James, Li-Hong Hu, Jia Li, and Ji-Ming Ye. Berberine and its more biologically available derivative, dihydroberberine, inhibit mitochondrial respiratory complex i. Diabetes, 57(5):1414-1418, 2008.

[44] Joseph Baur and David Sinclair. Therapeutic potential of resveratrol: The in vivo evidence. Nature reviews. Drug discovery, 5:493-506, 072006.

[45] Jee-Hyun Um, Sung-Jun Park, Hyeog Kang, Shutong Yang, Marc Foretz, Michael W. McBurney, Myung K. Kim, Benoit Viollet, and Jay H. Chung. Amp-activated protein kinase-deficient mice are resistant to the metabolic effects of resveratrol. Diabetes, 59(3):554-563, 2010.

[46] Nathan Price, Ana Gomes, Alvin J.Y. Ling, Filipe Duarte, Alejandro Martin-Montalvo, Brian North, Beamon Agarwal, Lan Ye, Giorgio Ramadori, João Teodoro, Basil Hubbard, Ana Varela, James Davis, Behzad Varamini, Angela Hafner, Ruin Moaddel, Anabela Rolo, Roberto Coppari, Carlos Palmeira, and David Sinclair. Sirt1 is required for ampk activation and the beneficial effects of resveratrol on mitochondrial function Cell metabolism, 15:675-90, 052012.

[47] Xiuyun Hou, Shanqin Xu, Karlene Maitland-Toolan, Kaori Sato, Bingbing Jiang, Yasuo Ido, Fan Lan, Kenneth Walsh, Michel Wierzbicki, Tony Verbeuren, Richard Cohen, and Mengwei Zang. Sirt1 regulates hepatocyte lipid metabolism through activating amp-activated protein kinase. The Journal of biological chemistry, 283:20015-26, 082008.

[48] Joseph Baur, Kevin Pearson, Nathan Price, Hamish Jamieson, Carles Lerin, Avash Kalra, Vinayakumar Prabhu, Joanne Allard, Guillermo Lopez-Lluch, Kaitlyn Lewis, Paul Pistell, Suresh Poosala, Kevin Becker, Olivier Boss, Dana Gwinn, Mingyi Wang, Sharan Ramaswamy, Kenneth Fishbein, Richard Spencer, and David Sinclair. Resveratrol improves health and survival of mice on a high-calorie diet. Nature, 444:337-342, 112006.

[49] Silvie Timmers, Ellen Konings, Lena Bilet, Riekelt Houtkooper, Tineke van de Weijer, Gijs Goossens, Joris Hoeks, Sophie Krieken, Dongryeol Ryu, Sander Kersten, Esther Moonen-Kornips, Matthijs Hesselink, Iris Kunz, Vera Schrauwen-Hinderling, Ellen Blaak, Johan Auwerx, and Patrick Schrauwen. Calorie restriction-like effects of 30 days of resveratrol supplementation on energy metabolism and metabolic profile in obese humans. Cell metabolism, 14:612-22, 112011

[50] Sanne van der Made, Jogchum Plat, and Ronald Mensink. Resveratrol does not influence metabolic risk markers related to cardiovascular health in overweight and slightly obese subjects: A randomized, placebo-controlled crossover trial. PloS one, 10:e0118393, 032015.

[51] Yueshui Zhao, Bo Chen, Jing Shen, Lin Wan, Yinxin Zhu, and Zhangang Xiao. The beneficial effects of quercetin, curcumin, and resveratrol in obesity. Oxidative Medicine and Cellular Longevity, 2017:1-8, 082017.

[52] Catalina Carrasco-Pozo, María Cires, and Martin Gotteland. Quercetin and epigallocatechin gallate in the prevention and treatment of obesity: From molecular to clinical studies. Journal of Medicinal Food, 22, 052019.

[53] Qu Collins, Hui-Yu Liu, Jingbo Pi, Zhenqi Liu, Michael Quon, and Wenhong Cao. Epigallocatechin-3-gallate (egcg), a green tea polyphenol, suppresses hepatic gluconeogenesis through 
5 '-amp-activated protein kinase. The Journal of biological chemistry, 282:30143-9, 102007.

[54] Kejing Zeng, Lili Tian, Rucha Patel, Weijuan Shao, Zhuolun Song, Ling Liu, Justin Manuel, Max Ma, Ian McGilvray, Carolyn Cummins, Jianping Weng, and Tianru Jin. Diet polyphenol curcumin stimulates hepatic fgf21 production and restores its sensitivity in high-fat-diet-fed male mice. Endocrinology, 158:jc.2016.1596, 122016.

[55] Jin Zhou, Benjamin Farah, Rohit Sinha, Yajun Wu, Brijesh Singh, Boon-Huat Bay, Chung Yang, and Paul Yen. Epigallocatechin-3-gallate (egcg), a green tea polyphenol, stimulates hepatic autophagy and lipid clearance. PloS one, 9:e87161, 012014.

[56] Liang Liu, Chao Gao, Ping Yao, and Zhiyong Gong. Quercetin alleviates high-fat diet-induced oxidized low-density lipoprotein accumulation in the liver: Implication for autophagy regulation. BioMed Research International, 2015:1-9, 122015.

[57] Naresh Kumar and Nidhi Goel. Phenolic acids: Natural versatile molecules with promising therapeutic applications. Biotechnology reports (Amsterdam, Netherlands), 24:e00370e00370, Aug 2019. 31516850[pmid].

[58] Gregory R. Steinberg, Madhumita Dandapani, and D. Grahame Hardie. Ampk: mediating the metabolic effects of salicylate-based drugs? Trends in Endocrinology and Metabolism, 24(10):481-487, October 2013. G.R.S. is supported by a Canada Research Chair in Metabolism and Obesity and grants from the Canadian Institutes of Health Research and the Canadian Diabetes Association. D.G.H. is supported by a Senior Investigator Award from the Wellcome Trust and a Programme Grant from Cancer Research UK, and by the pharmaceutical companies supporting the Division of Signal Transduction Therapy at Dundee (AstraZeneca, Boehringer-Ingelheim, GlaxoSmithKline, Merck KgaA, Janssen Pharmaceutica, and Pfizer). M.D. was supported by a Clinical PhD studentship from the Wellcome Trust. Commissioned review article.

[59] JR Vane. Inhibition of prostaglandin synthesis as a mechanism of action for aspirin-like drugs. Nature: New biology, 231(25):232-235, June 1971.

[60] E Kopp and S Ghosh. Inhibition of nf-kappa b by sodium salicylate and aspirin. Science (New York, N.Y.), 265(5174):956-959, August 1994.

[61] Carmen Montesinos and Gerald Weissmann. Salicylates and sulfasalazine, but not glucocorticoids, inhibit leukocyte accumulation by an adenosine-dependent mechanism that is independent of inhibition of prostaglandin synthesis and p105 of nfkappa b. Proceedings of the National Academy of Sciences, 96, 061999.

[62] Simon A. Hawley, Morgan D. Fullerton, Fiona A. Ross, Jonathan D. Schertzer, Cyrille Chevtzoff, Katherine J. Walker, Mark W. Peggie, Darya Zibrova, Kevin A. Green, Kirsty J. Mustard, Bruce E. Kemp, Kei Sakamoto, Gregory R. Steinberg, and D. Grahame Hardie. The ancient drug salicylate directly activates amp-activated protein kinase. Science (New York, N.Y.), 336(6083):918-922, May 2012. 22517326[pmid].

[63] Andrew Obrien, Linda Villani, Lindsay Broadfield, Vanessa Houde, Sandra Galic, Giovanni Blandino, Bruce Kemp, Theodoros Tsakiridis, Paola Muti, and Gregory Steinberg. Salicylate activates ampk and synergizes with metformin to reduce the survival of prostate and lung cancer cells ex vivo through inhibition of de novo lipogenesis. The Biochemical journal, 469, 052015.

[64] Lin Han, Qing Yang, Jia Li, feier cheng, Yao Zhang, Yunlong
$\mathrm{Li}$, and Min Wang. Protocatechuic acid-ameliorated endothelial oxidative stress through regulating acetylation level via cd36/ampk pathway. Journal of Agricultural and Food Chemistry, 67, 062019.

[65] Yunu Jung, Jinbong Park, Hye-Lin Kim, Jung-Eun Sim, DongHyun Youn, Jongwook Kang, Seona Lim, Mi-Young Jeong, Woong Mo Yang, Seok-Geun Lee, Kwang Ahn, and Jae-Young Um. Vanillic acid attenuates obesity via activation of the ampk pathway and thermogenic factors in vivo and in vitro. The FASEB Journal, 32:fj.201700231RR, 112017.

[66] Miori Tanaka, Akari Sato, Yoshimi Kishimoto, Hideaki Mabashi-Asazuma, Kazuo Kondo, and Kaoruko Iida. Gallic acid inhibits lipid accumulation via ampk pathway and suppresses apoptosis and macrophage-mediated inflammation in hepatocytes. Nutrients, 12(5), May 2020.

[67] Khanh Doan, Chang Ko, Ann Kinyua, Dong Yang, Yun-Hee Choi, In Oh, Nguyen Nguyen, Ara Ko, Jae Choi, Yangsik Jeong, Min Jung, Won Cho, Shanhua Xu, Kyu-Sang Park, Woo Park, Soo Choi, Hyoung Kim, Sang Hyun Moh, and Ki Kim. Gallic acid regulates body weight and glucose homeostasis through ampk activation. Endocrinology, 156:en20141354, 10 2014.

[68] En-Pei Chiang, Shu-Yao Tsai, Yueh-Hsiung Kuo, Man-Hui Pai, Hsi-Lin Chiu, Raymond Rodriguez, and Feng-Yao Tang. Caffeic acid derivatives inhibit the growth of colon cancer: Involvement of the pi3-k/akt and ampk signaling pathways. PloS one, 9:e99631, 062014.

[69] Rafaela Scalco Ferreira, Neife Santos, Carolina Bernardes, Flávia Malvestio Sisti, Lilian Amaral, Andréia Fontana, and Antonio Santos. Caffeic acid phenethyl ester (cape) protects pc12 cells against cisplatin-induced neurotoxicity by activating the ampk/sirt1, mapk/erk, and pi3k/akt signaling pathways. Neurotoxicity Research, 36, 042019.

[70] Eun Lee, Kyung-Ok Uhm, Yun Lee, Myoung Sook Han, MyungSik Lee, Ji-Man Park, Pann-Ghill Suh, Sun-Hwa Park, and Hyeon Kim. Cape (caffeic acid phenethyl ester) stimulates glucose uptake through ampk (amp-activated protein kinase) activation in skeletal muscle cells. Biochemical and biophysical research communications, 361:854-8, 112007.

[71] Hoda Eid, Farah Thong, Abir Nachar, and Pierre Haddad. Caffeic acid methyl and ethyl esters exert potential antidiabetic effects on glucose and lipid metabolism in cultured murine insulin-sensitive cells through mechanisms implicating activation of ampk. Pharmaceutical Biology, 55:2026-2034, 122017.

[72] Xiaoling Chen, Yafei Guo, Gang Jia, Hua Zhao, Guangmang Liu, and Zhiqing Huang. Ferulic acid regulates muscle fiber type formation through sirt1/ampk signaling pathway. Food Function, 10, 112018.

[73] Seung-Woo Kang, Seong-Il Kang, Hye-Sun Shin, Seon-A Yoon, Jeong-Hwan Kim, Hee-Chul Ko, and Se-Jae Kim. Sasa quelpaertensis nakai extract and its constituent p-coumaric acid inhibit adipogenesis in 3t3-11 cells through activation of the ampk pathway. Food and chemical toxicology : an international journal published for the British Industrial Biological Research Association, 59, 062013. 